\title{
Twenty-four-hour urinary sodium and potassium excretion and associated factors in Japanese secondary school students: methodological issues that can lead to misinterpretation
}

\author{
Hypertension Research (2017) 40, 625; doi:10.1038/hr.2016.186; published online 19 January 2017
}

We were interested to read the article by Okuda et al. ${ }^{1}$ that was published in Hypertension Research in July 2016. The authors aimed to evaluate the effects of height, weight, age, spot urine mineral concentration, maturation and physical activity on 24-h sodium and potassium excretion in Japanese secondary school students. The authors noted in their conclusion that physical activity is an important determinant of 24-h sodium and potassium excretion in adolescents. ${ }^{1}$ Such a conclusion can be considered optimistic, unless the result can be validated internally or externally using bootstrap reputation, split validation and cross-validation. ${ }^{2}$

Regression models of 24-h sodium and potassium excretion showed small regression co-efficients for the explanatory variables and large intercepts. The intercept will be large if the number of observations and the number of explanatory variables are low. The sample size in the regression analysis by Okuda et al. ${ }^{1}$ was sufficient, as 320 secondary school adolescents were recruited for the study. It seems that the large intercept was a result of neglecting other relevant determinants of 24-h sodium and potassium excretion. Given that the authors reported important determinants of 24 -h sodium and potassium excretion in adolescents, such a conclusion should be interpreted with caution.

Moreover, the authors should have reported the confidence intervals for the estimated associations. Reporting only $p$-values is a common mistake. ${ }^{3}$

\section{CONFLICT OF INTEREST}

The authors declare no conflict of interest.

Erfan Ayubi ${ }^{1,2}$, Mohadeseh Sani ${ }^{3}$, Kamyar Mansori ${ }^{4,5}$ and Salman Khazaei ${ }^{6}$

${ }^{1}$ Department of Epidemiology, School of Public Health, Shahid Beheshti University of Medical Sciences, Tehran, Iran; ${ }^{2}$ Department of Epidemiology \& Biostatistics, School of
Public Health, Tehran University of Medical Sciences, Tehran, Iran; ${ }^{3}$ School of Medicine, Zabol University of Medical Sciences, Zabol, Iran; ${ }^{4}$ Social Determinants of Health Research Center, Kurdistan University of Medical Sciences, Sanandaj, Iran;

${ }^{5}$ Department of Epidemiology, School of Public Health, Iran University of Medical Sciences, Tehran, Iran and ${ }^{6}$ Department of Epidemiology, School of Public Health, Hamadan University of Medical Sciences, Hamadan, Iran E-mail: salman.khazaei61@gmail.com

1 Okuda M, Asakura K, Sasaki S, Shinozaki K. Twenty-four-hour urinary sodium and potassium excretion and associated factors in Japanese secondary school students. Hypertens Res 2016; 39: 524-529.

2 Steyerberg E. Clinical Prediction Models: A Practical Approach to Development, Validation, and Updating. Springer Science \& Business Media: New York, USA, 2008.

3 Rothman KJ, Greenland S, Lash TL. Modern epidemiology. Lippincott Williams \& Wilkins: Philadelphia, USA, 2008. 